\title{
The principle of crater-formation of a concrete target plate penetrated by a projectile
}

\author{
G. Shiqiao, J. Lei, L. Haipeng \& L. Kejie \\ School of Mechatronic Engineering, Beijing Institute of Technology, \\ People's Republic of China
}

\begin{abstract}
Based on a series of experimental and theoretical analyses for a concrete target penetrated by a projectile, a 4-stage model of crater-forming is presented in which the forming process of a crater is divided into 4 stages. Based on the Normal Expansion Theory, the analytical expressions of resistant forces on the projectile are given. Through analysing tensile fracture and considering dynamical cracks bifurcation and propagation, the sizes of craters are calculated. They are in good agreement with the test results.
\end{abstract}

Keywords: crater, concrete target, penetration, projectile.

\section{Introduction}

Many efforts have been made in the past 100 years to predict the dynamic characteristic of a projectile impacting, penetrating, perforating and damaging a concrete target [1-4], but a few are about the formation of a crater on the front surface of the target. When a concrete target plate with brittle material behavior is penetrated by a projectile, an unavoidable crater on its front surface will occur. The sizes of the crater depend usually on the striking velocity, the shape and sizes of projectile nose, and the mechanical behavior of the concrete material. The forming process of a crater is the initial sub-process among the entire penetration process. It is very difficult to analyze in mechanics. On one hand, the forming process of a crater is an important sub-process of the total penetration process. On the other hand, the formation of crater is too complex to accurately evaluate by general analytical or numerical methods. Up to now, the principle of crater-forming has not been well explained. The expressed formula of resistant forces of target on the projectile in this sub-process has not been analytically 
obtained. Based on some post-test observations of soil and concrete target $[4,5]$, Forrestal et al. [3] gave an approximate empirical resistant force model during the crater process. Forrestal et al. pointed out that, the cavity after penetration is a conical region with length about two projectile shank diameter, followed by a circular cylinder with diameter nearly equal to the projectile shank diameter. But they didn't answer how the crater is formed. To understand how the crater is formed, how great the crater is, and which parameters it concerns, it is very necessary and important to study the principle of the crater forming.

Based on a series of experimental and theoretical analyses (including contact and crack mechanics) of a concrete target penetrated by a projectile, a 4-stage model of crater-forming is presented, in which the forming process of a crater is divided into 4 stages. The first stage is elastic contact stage, during which the concrete target undergoes the elastic deformation under the pressure of rigid projectile nose. The second stage is the radial fracture stage during which a series of circumferential cracks are formed and grow on the free surface of concrete target. At the same time, in front region of the nose tip, many crossed cracks are formed, and near the surface of the projectile nose, the concrete medium will be crushed. The third stage is the circumferential fracture stage during which a series of radial cracks are formed and grow so that many crossed cracks (crack group) are formed near the free surface of target, which result in many fragments. Under the pressure of projectile nose with high striking velocity, these fragments are thrown out. At the same time, the crushed medium near the surface of projectile nose will be expanded. The crossed cracks in front of the nose tip of projectile will grow so that a crack nucleation is formed. The fourth stage is the crater-forming stage during which both the crossed cracks near the free surface of target and the crack nucleation grow so rapidly as to encounter each other to form the crater.

\section{Principle of crater-forming based on the force analyses in theory}

During the penetration of nose tip of projectile into the semi-infinite concrete target, the medium near the surface of projectile nose will be crushed. The stresses distribution of the medium far from the projectile nose is equivalent to that caused by a concentrated compressive force load. When a concentrated compressive load is applied on the free surface of semi-infinite body, the stress distributions are shown in Fig.1. From Fig.1, the following phenomena can be seen. 1). Radial stresses shown in Fig.1a are tensile both near the free surface and near the symmetrical axis, but are compressive in other regions. 2). Circumferential stresses shown in Fig.1b are tensile only in the region near the symmetrical axis, but are compressive in other regions including the free surface. 3). Axial stresses shown in Fig.1c are compressive in all the regions. One of the most obvious behaviors of concrete material is that the tensile strength is much smaller than its compressive strength. Therefore the cause of crater-forming is thought of as the tensile fractures. During the first stage, that is, the initial penetration, the applied force between the target and the projectile is 
relatively small, the concrete material is subject to the elastic deformation. With the growth of the force between target and projectile, the compressive stresses near the nose tip of the projectile increase so rapidly as to reach the compressive strength and to crush the medium in this region. At the same time, the circumferential cracks start occurring in the medium near the free surface (not only near the nose of projectile, but also far from it). A series of conical tubes are formed because of the radial stress beyond the tensile strength. This is the second stage. During the second stage, the medium near the symmetrical axis is also fractured by both the action of radial and circumferential tensile stresses. Many crossed cracks are formed. With the incessant growth of the mutual interactive force, the crack regions are incessantly widened (propagated). With the deeper penetration of projectile, by the action of radial expansive pressure of projectile nose, the circumferential stresses of formed conical tubes will reach the level of tensile strength to form a series of radial cracks. This is the third stage. During this stage, the crossed cracks in front of nose tip of projectile incessantly grow to form a crack nucleation. Up to now, two crack group regions are formed. One is near to the free surface of target. The other is near to the symmetrical axis. On both boundary surfaces of crack regions there are many crack tips. For dynamic fracture of a brittle concrete material, one of the peculiar behaviors is that the cracks tend to branch out (bifurcate) in order to decrease the overall energy of the system at a certain velocity. These bifurcations grow toward the weak regions and result in the encounter of the two cracks regions to form a crater. A schematic crater formation is shown in Fig.2. The fractured medium in the crater is thrown out in the form of fragments due to the lateral velocity of projectile nose surface.

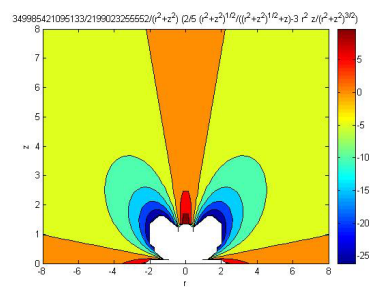

(a)

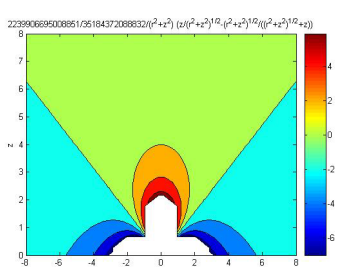

(b)

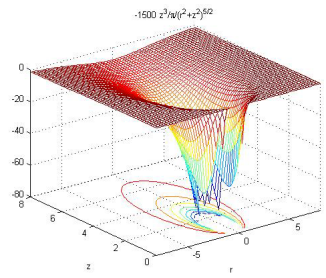

(c)

Figure 1: The distributions of stresses of a semi-infinite body by pressure of a concentrated force.

\section{The resistant forces during the crater penetration}

According to the Normal Expansion Theory (NET) [6-10], the resistant forces applied on the projectile nose are mainly composed of two portions. One comes from the strength effect. The other comes from the inertia effect. The strength implies the compressive strength under hydrostatic pressures. The inertia implies the mass increasing of the responding medium with acceleration. 
During the first elastic stage, based on the solutions of contact mechanics, the pressure $\mathrm{P}$ can be expressed as

$$
P=\frac{4 E \sqrt{R}}{3\left(1-\mu^{2}\right)} x^{3 / 2}
$$

where $E$ is Young's modulus of the target material, $R$ is the radius of nose tip of the projectile, $\mu$ is Poisson's ratio of the target material, $x$ is the depth of penetration.

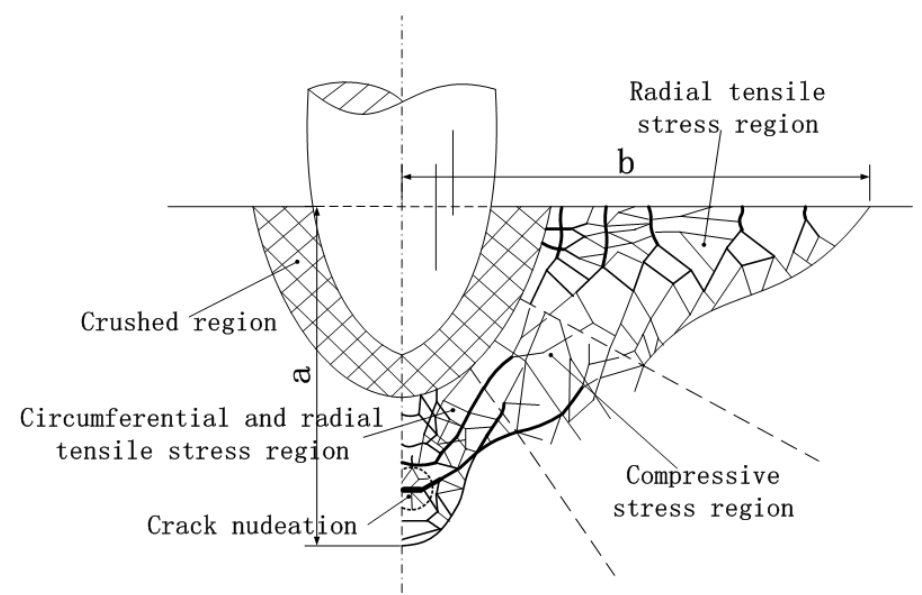

Figure 2: The schematic crater formation.

During the rest stages, the axial pressure can be written $[6,10]$ as

$$
P=\iint_{S_{A}} \sigma_{c} \cos \varphi d s+\frac{m_{p}}{m_{p}+m_{f}} \iint_{S_{A}} \frac{\rho^{*} \rho_{0}}{\rho^{*}-\rho_{0}} v^{2} \cos ^{3} \varphi d s
$$

where $m_{f}=\iint_{S_{A}} \rho^{*} l \cos ^{2} \varphi d s$ is additional mass of responding medium, $\sigma_{c}$ is the compressive strength under high strain rate, $\varphi$ is a coordinate describing the angle between the normal of nose surface of projectile and symmetrical axis, $m_{p}$ is the mass of projectile, $\rho_{0}$ is the density of concrete target material, $\rho^{*}$ is the ultimate density of concrete target material, $v$ is the instantaneous velocity of projectile, $l$ is the propagating distance of the responding medium wave, $S_{A}$ is the curve surface portion of projectile nose which has penetrated into the target.

In the right hand side of equation (2), the first term is the strength term and the second term is the inertia term. 


\section{The estimation of the size of crater}

Near the surface of projectile nose, the concrete medium is compressed by so high pressure as to reach the compressive strength. The density of this region increases so rapidly as to reach the ultimate level, which has been discussed by Gao et al. [6-10]. Far from the projectile nose, the distributions are the same as those caused by concentrated force. In terms of the analyses in section 2 , there are two cracks regions, one near to the free surface of target, the other near to the symmetrical axis.

The stress distributions of a semi-infinite object pressed by a concentrated load can be expressed as

$$
\left\{\begin{array}{c}
\sigma_{z}=-\frac{3}{2} \frac{P}{\pi r^{2}} \cos ^{3} \varphi \\
\sigma_{r}=\frac{1}{2} \frac{P}{\pi r^{2}}\left[(1-2 \mu) \frac{1}{1+\cos \varphi}-3 \cos \varphi \sin ^{2} \varphi\right] \\
\sigma_{\theta}=\frac{1}{2} \frac{P}{\pi r^{2}}(1-2 \mu)\left(\cos \varphi-\frac{1}{1+\cos \varphi}\right)
\end{array}\right.
$$

where $\sigma_{z}, \sigma_{r}$ and $\sigma_{\theta}$ are axial, radial and circumferential stresses respectively, $P$ is the concentrated load, $r$ is the radial coordinate, $\varphi$ is the angular coordinate, $\mu$ is Poisson's ratio of the concrete material.

Near to the free surface, the radial stress is tensile stress. It can be written as

$$
\sigma_{r}=\frac{1-2 \mu}{2 \pi b^{2}} P
$$

where $b$ is the radial distance.

Near to the symmetrical axis, the circumferential stress can be written as

$$
\sigma_{\theta}=\frac{1-2 \mu}{4 \pi a^{2}} P
$$

where $a$ is the axial distance. In the axis, the radial stress is equal to the circumferential stress. Both are tensile.

According to the analyses of crack formation mentioned above, whether radial stress $\sigma_{r}$ or circumferential stress $\sigma_{\theta}$ reach the level of tensile strength $\sigma_{t}$ of the target material, some cracks will be formed. Near the free surface, because of a non-restrained boundary, all the cracks will be easy to grow. Whereas, near the symmetrical axis, because of the restraint of compressive stress around the crack region, not all cracks can grow, but only the cracks whose tensile stress reaches a new level can grow to form a nucleation. This new stress level can be written as $k \sigma_{t}$, where $k(>1)$ is a parameter of forming 
crack nucleation. Substituting $\sigma_{r}=\sigma_{t}$ and $\sigma_{\theta}=k \sigma_{t}$ into equation (4) and (5), yields

$$
\begin{aligned}
& b=\sqrt{\frac{1-2 \mu}{2 \pi} \frac{P}{\sigma_{t}}} \\
& a=\sqrt{\frac{1-2 \mu}{4 k \pi} \frac{P}{\sigma_{t}}} \\
& \operatorname{tg} \alpha=\frac{a}{b}=\frac{1}{\sqrt{2 k}}
\end{aligned}
$$

where $\alpha$ is called crater angle.

From equation (8), it can be obviously seen that, the crater angle is independent either of the pressure or of the tensile strength.

\section{Tests results in the shot field}

In the past three years, a series of field tests were conducted for obtaining the deceleration curves of a projectile penetrating concrete targets [7,8]. At the same time, many geometric data have been obtained about the crater on the front surface and the scabbing on the rear surface of the target plates. It is obvious from the tests that, after penetration there is a nearly conical crater on the front surface of concrete target plate.

We conducted every test with three target plates as a combination target shown in Fig.4. The distance between two plates is $1 \mathrm{~m}$. The diameter of the target plate is $1.5 \mathrm{~m}$. Except the first plate in the first test whose thickness is $300 \mathrm{~mm}$, all other plates have $200 \mathrm{~mm}$ thickness. The projectile has $3.0 \mathrm{~kg}$ mass, $62 \mathrm{~mm}$ shank diameter, $75 \mathrm{~mm}$ ogive radius and $1.21 \mathrm{CRH}$ (caliber-radius-head), shown in Fig.3. An example of the crater after penetration is shown in Fig.5 and Fig.6.

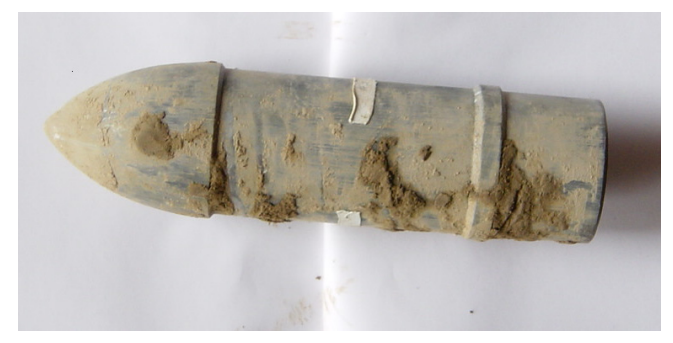

Figure 3: $\quad$ The projectile used in the tests.

Some data about the sizes of craters after penetration are listed in Tab.1 where $v_{s}$ is the striking velocity of projectile, $f_{c}^{\prime}$ is unconfined compressive strength, 
$S_{d}$ is the depth of crater, $D_{c}$ is the diameter of crater, $D_{s}$ is the diameter of scabbing, $d_{p}$ is the diameter of perforated tunnel.

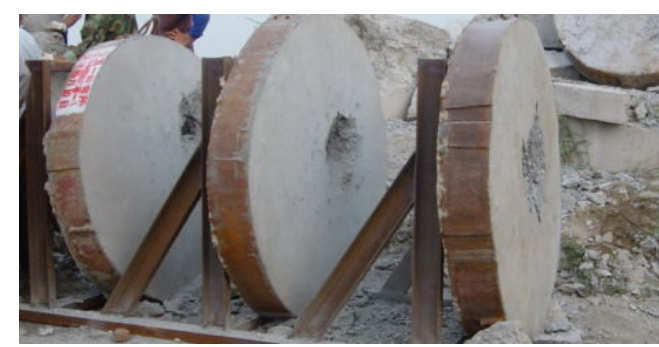

Figure 4: The combination target consisting of three concrete plates.

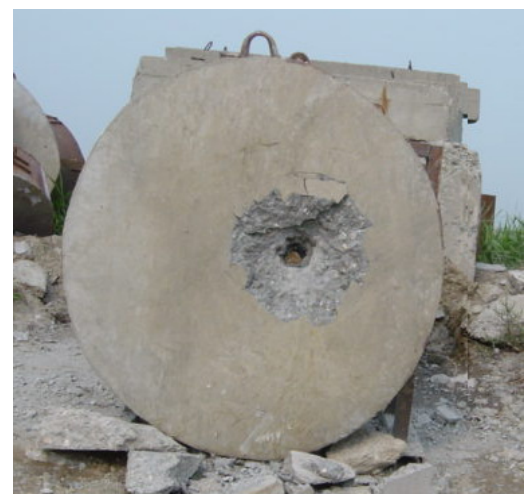

Figure 5: The crater on the front surface crater of target plate after penetration.

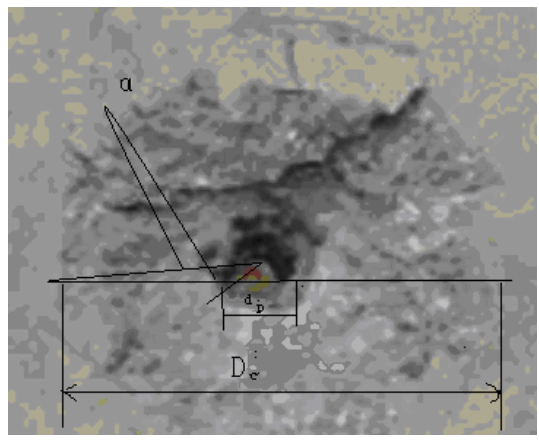

Figure 6: The size description of the crater. 
Table 1: Data summary about sizes of crater, scabbing and tunnel after penetration.

\begin{tabular}{|c|c|c|c|c|c|c|c|}
\hline $\begin{array}{l}\text { The } \\
\text { shot } \\
\text { number }\end{array}$ & $\begin{array}{l}v_{s} \\
(\mathrm{~m} / \mathrm{s})\end{array}$ & $\begin{array}{l}\text { Target } \\
\text { plate } \\
\text { number }\end{array}$ & $\begin{array}{l}f_{c}^{\prime} \\
\mathrm{Mpa}\end{array}$ & $\begin{array}{l}S_{d} \\
(\mathrm{~mm})\end{array}$ & $\begin{array}{l}D_{c} \\
(\mathrm{~mm})\end{array}$ & $\begin{array}{l}D_{s} \\
(\mathrm{~mm})\end{array}$ & $\begin{array}{l}d_{p} \\
(\mathrm{~mm})\end{array}$ \\
\hline \multirow{3}{*}{1} & \multirow{3}{*}{590} & 1 & 45 & 150 & $550-600$ & $460-700$ & 100 \\
\hline & & 2 & 35 & 110 & $350-430$ & $480-600$ & 150 \\
\hline & & 3 & 30 & - & - & - & - \\
\hline \multirow{3}{*}{2} & \multirow{3}{*}{670} & 1 & 35 & 100 & $410-570$ & $500-520$ & 160 \\
\hline & & 2 & 35 & 80 & $380-420$ & $490-550$ & 100 \\
\hline & & 3 & 30 & 90 & $360-430$ & $550-850$ & 140 \\
\hline \multirow{3}{*}{3} & \multirow{3}{*}{669} & 1 & 35 & 110 & $410-510$ & $450-550$ & 140 \\
\hline & & 2 & 35 & 90 & $450-550$ & $460-520$ & 100 \\
\hline & & 3 & 30 & 90 & $300-450$ & $480-610$ & 100 \\
\hline \multirow{3}{*}{4} & \multirow{3}{*}{653} & 1 & 35 & 100 & $400-430$ & 500 & 130 \\
\hline & & 2 & 30 & 90 & $320-440$ & $540-620$ & 120 \\
\hline & & 3 & 30 & 85 & $360-410$ & $440-500$ & 80 \\
\hline \multirow{3}{*}{5} & 650 & 1 & 45 & 150 & - & $600-800$ & 130 \\
\hline & & 2 & 30 & - & 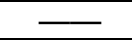 & - & - \\
\hline & & 3 & 30 & - & - & - & - \\
\hline
\end{tabular}

\section{An example}

From the test results, the curve of crater angles versus different shots is shown in Fig.7. It can be obviously seen that the crater angles vary not obviously either with the striking velocities, or with the compressive strengths of material. This has validated the equation (8). From the statistics of test results, the mean value of crater angle is $24.7^{0}$. The parameter $k$ can be obtained as 2.36 .

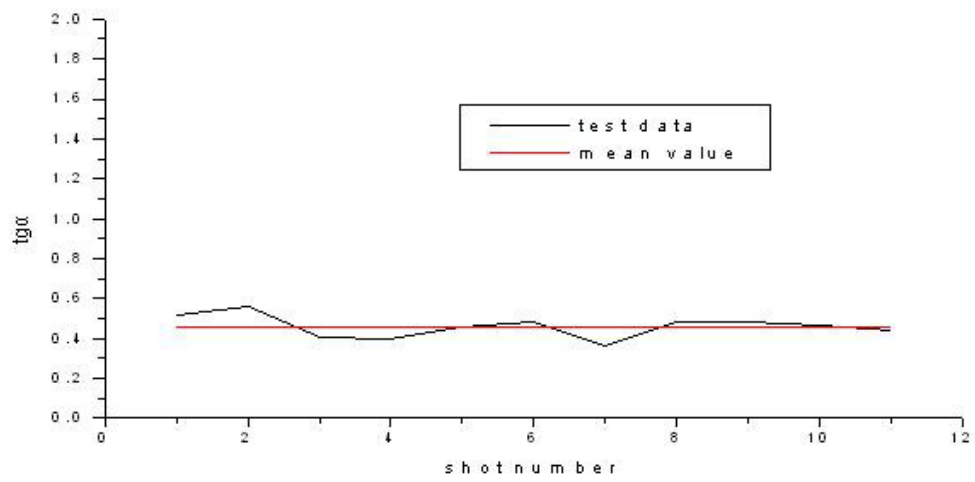

Figure 7: The curve of crater angle vs. shot number and the mean value. 
As an example, the sizes of crater have been calculated. The penetrating projectile has $3.0 \mathrm{~kg}$ mass, $62 \mathrm{~mm}$ shank diameter, $75 \mathrm{~mm}$ ogive radius and 1.21 CRH (caliber-radius-head). The concrete target material has $3 \mathrm{Mpa}$ tensile strength, $35 \mathrm{Mpa}$ compressive strength, and $2400 \mathrm{~kg} / \mathrm{m} 3$ density. For $670 \mathrm{~m} / \mathrm{s}$ striking velocity, the calculated crater diameter is $0.42 \mathrm{~m}$. For $653 \mathrm{~m} / \mathrm{s}$ striking velocity, the calculated crater diameter is $0.4 \mathrm{~m}$. They are in good agreement with the results from tests.

\section{Acknowledgements}

This work is supported by the BIT Constract-51309. The first author would like to acknowledge Alexander von Humboldt Foundation for her support when stayed with Mr. Dr.-Ing. Michael Kasperski in Faculty of Civil Engineering of Ruhr University Bochum in Germany.

\section{Reference}

[1] Bacckmann, M.E., Goldsmith, W., The mechanics of projectiles into target. Int J Engng Sci, 1978; 16:1-99.

[2] Kennedy, R. P., A review of procedures for the analysis and design of concrete structures to resist missile impact effect. Nuclear Engineering and Design. 1976; 37: 183-203.

[3] Forrestal, M. J., Altman, B. S., Cargile, J. D. and Hanchak, S. J., An empirical equation for penetration depth of ogive-nose projectiles into concrete targets. International Journal of Impact Engineering. 1994, 15, 395-405.

[4] Forrestal, M. J. and Luk, V. K., Penetration into soil target. International Journal of Impact Engineering, 1992, 12, 427-444.

[5] Forrestal, M. J., Brar, N. S. and Luk, V. K., Penetration of strainhardening targets with rigid spherical-nose rods. ASME Journal of Applied mechanics. 1991, 58, 7-10. H. Kolsky, Stress waves in solids, Dover, New York, 1963.

[6] Gao Shiqiao, Jin Lei \& Liu Haipeng, Dynamic response of a projectile perforating multi-plate concrete targets. Int J Solids and Structures, 2004; 41: 4927-4938.

[7] Gao Shiqiao, Liu Mingjie, \& Tan Huimin. Dynamic analysis of a penetrator penetrating against half-infinite concrete target, ACTA Amarmenterii, 1995; 16(4): 46-50.

[8] Liu Haipeng, Gao Shiqiao and Li Kejie Measurement technologies and result analysis on experiment of penetration of steel projectile into thick concrete target, Proc. of 5th International Conference of Measurements and Tests, 2003.

[9] Gao Shiqiao, Shi Gengchen, Tan Huimin and Guo Zhaoping. Dynamic analysis of Projectile-fuze system impacting half-infinite concrete target with small fall-angle, Journal of Beijing institute of Technology,1994; 14(4): 359-365. 
322 Structures Under Shock and Impact IX

[10] Gao Shiqiao, Liu Haipeng, Li Kejie, Huang Fenglei, Jin Lei. Normal Expansion Theory for Penetration of a Projectile against Concrete Target. Applied Mathematics and Mechanics. 2006; 27 (4): 485-492. 